\title{
Nonuniversal scaling of the magnetocaloric effect as an insight into spin-lattice interactions in manganites
}

Smith, Anders; Nielsen, Kaspar Kirstein; Neves Bez, Henrique; Bahl, Christian

Published in:

Physical Review B

Link to article, DOI:

10.1103/PhysRevB.94.054411

Publication date:

2016

Document Version

Publisher's PDF, also known as Version of record

Link back to DTU Orbit

Citation (APA):

Smith, A., Nielsen, K. K., Neves Bez, H., \& Bahl, C. (2016). Nonuniversal scaling of the magnetocaloric effect as an insight into spin-lattice interactions in manganites. Physical Review B, 94(5), [054411].

https://doi.org/10.1103/PhysRevB.94.054411

\section{General rights}

Copyright and moral rights for the publications made accessible in the public portal are retained by the authors and/or other copyright owners and it is a condition of accessing publications that users recognise and abide by the legal requirements associated with these rights.

- Users may download and print one copy of any publication from the public portal for the purpose of private study or research.

- You may not further distribute the material or use it for any profit-making activity or commercial gain

- You may freely distribute the URL identifying the publication in the public portal 


\title{
Nonuniversal scaling of the magnetocaloric effect as an insight into spin-lattice interactions in manganites
}

\author{
Anders Smith, ${ }^{*}$ Kaspar K. Nielsen, Henrique N. Bez, and Christian R. H. Bahl \\ Department for Energy Conversion and Storage, Technical University of Denmark, Ris $\varnothing$ Campus, \\ Frederiksborgvej 399, DK-4000 Roskilde, Denmark \\ (Received 19 October 2015; published 9 August 2016)
}

\begin{abstract}
We measure the magnetocaloric effect of the manganite series $\mathrm{La}_{0.67} \mathrm{Ca}_{0.33-x} \mathrm{Sr}_{x} \mathrm{MnO}_{3}$ by determining the isothermal entropy change upon magnetization, using variable-field calorimetry. The results demonstrate that the field dependence of the magnetocaloric effect close to the critical temperature is not given uniquely by the critical exponents of the ferromagnetic-paramagnetic phase transition, i.e., the scaling is nonuniversal. A theoretical description based on the Bean-Rodbell model and taking into account compositional inhomogeneities is shown to be able to account for the observed field dependence. In this way the determination of the nonuniversal field dependence of the magnetocaloric effect close to a phase transition can be used as a method to gain insight into the strength of the spin-lattice interactions of magnetic materials. The approach is shown also to be applicable to first-order transitions.
\end{abstract}

DOI: 10.1103/PhysRevB.94.054411

For the past several decades manganites have been a rich field of study for their many fascinating properties, including colossal magnetoresistance, multiferroic effects, and charge and orbital ordering leading to inhomogenous phases such as stripes [1-3]. In recent years several manganites have also been studied for their magnetocaloric properties, both in bulk [4,5] and in thin films, where it was shown that the effect of substrate strain can increase the magnetocaloric effect significantly [6]. This has been motivated by their possible application in near-room-temperature magnetic refrigeration [7]. However, measurements of the magnetocaloric effect can also give important information on the intrinsic magnetic properties of materials, as has been realized since the very discovery of the effect by Weiss and Piccard [8,9]. Of particular interest is the field dependence of the magnetocaloric quantities, which has been discussed extensively in recent literature. It has been claimed that the field dependence close to the critical temperature of the ferromagnetic-to-paramagnetic transition is universal and determined by the critical exponents of the system in question $[10,11]$. Recently we showed theoretically that the scaling of the magnetocaloric quantities is not universal: models in the same universality class differ in their scaling exponents at finite fields [12]. Here we show that this lack of universality is, in fact, observable experimentally and can be used to gain insight into the strength of the spin-lattice interactions, which ultimately are responsible for the appearance of the magnetocaloric effect.

The rare-earth manganites are in many ways an ideal system to study the field dependence of the magnetocaloric quantities, due to the ability of changing the critical temperature of the sample by only slight variations in the composition. The parent composition is the perovskite structure $\mathrm{AMnO}_{3}$, where A is a rare earth such as La. By substituting alkali metal or alkaline earth-metal cations on the A-site, one obtains ferromagnetic compounds with a critical temperature that varies strongly with composition. Here we study the series $\mathrm{La}_{0.67} \mathrm{Ca}_{0.33-x} \mathrm{Sr}_{x} \mathrm{MnO}_{3}$. When $x$ is varied from 0 to 0.33 ,

*Corresponding author: ansm@dtu.dk the critical temperature of the compound varies from $267 \mathrm{~K}$ to $369 \mathrm{~K}$ [13]. The universality class of these compounds has been investigated by a number of authors. Their findings are not in total agreement. Thus, several authors have found that one end member of the series, $\mathrm{La}_{0.67} \mathrm{Ca}_{0.33} \mathrm{MnO}_{3}$, has a first-order transition [14-16], but this has been questioned by detailed examination of the latent heat associated with the transition [17]. For the other end member, $\mathrm{La}_{0.67} \mathrm{Sr}_{0.33} \mathrm{MnO}_{3}$, there is agreement that the transition is second order, but the critical exponents have variously been determined as being those of the 3D Ising model $[18,19]$ and those of the 3D Heisenberg model [20,21]. For the series as a whole, no general studies of the universality class exist. Starting with $\mathrm{La}_{0.67} \mathrm{Sr}_{0.33} \mathrm{MnO}_{3}$ the main effect of substituting $\mathrm{Ca}$ for $\mathrm{Sr}$ is to change the $\mathrm{Mn}-\mathrm{O}-\mathrm{Mn}$ bond angle from $\sim 170^{\circ}$ to $\sim 162^{\circ}$, which changes the hopping integrals between the orbitals $\mathrm{e}_{\mathrm{g}}(\mathrm{Mn})-\mathrm{p}_{\sigma}(\mathrm{O})-\mathrm{e}_{\mathrm{g}}(\mathrm{Mn})$, causing a change in the coupling between the Mn spins [22,23]. The consensus seems to be that all the materials of the series except possibly close to $\mathrm{La}_{0.67} \mathrm{Ca}_{0.33} \mathrm{MnO}_{3}$ (i.e., small $x$ ) are in fact second order and of the same universality class. Even though the series has not been investigated extensively enough to rule out a crossover from one universality class to another (e.g., from 3D Heisenberg to 3D Ising) as $x$ is varied, it can be safely assumed that the critical exponents do not vary continuously with $x$.

The identification of the universality class is often done by determining the critical exponents $\beta$ and $\gamma$ (associated, respectively, with the scaling of the magnetization $M$ below $T_{c}$ and the scaling of the inverse susceptibility $\chi^{-1}$ above $T_{c}$ ) from magnetization data. A widely used procedure is that of Kaul [24,25]. From a starting guess of the critical exponents $\beta$ and $\gamma$ the modified Arrott plot of $M^{\beta}$ as a function of $(H / M)^{1 / \gamma}$ is constructed; here $H$ is the internal field (i.e., the applied field corrected for demagnetization). From the intersection of the curves (suitably extrapolated) with the coordinate axes, $M(T, H=0)$ and $\chi^{-1}(T, H=0)$ are extracted and new exponents $\beta^{\prime}$ and $\gamma^{\prime}$ calculated. The procedure is then repeated until convergence; $T_{c}$ is determined as the temperature corresponding to the $M^{\beta}$ versus $(H / M)^{1 / \gamma}$ curve that intersects $(0,0)$ when extrapolated. Although in principle rather 
straightforward, in practice this procedure requires a temperature resolution of the magnetization that is often not available: If, as is usual, magnetization curves are only recorded with a temperature step of 2-3 K, the number of curves that actually fall within the critical region is very limited. With a $T_{c}$ near room temperature a critical region of the order of $|t| \lesssim 10^{-2}$ [24] (where $t=\left(T-T_{c}\right) / T_{c}$ is the reduced temperature) corresponds to a temperature interval of 5-10 K. This means that only 4-5 Arrott curves fall within the critical region. To use these curves to extract three parameters $\left(\beta, \gamma, T_{c}\right)$ is clearly associated with considerable uncertainty. And since the critical exponents of, e.g., the 3D Heisenberg and 3D Ising models are in fact rather close, assigning a definite universality class on the basis of this procedure is often not warranted.

The analysis is further hampered by the fact that these systems have a marked tendency to form magnetically inhomogeneous states, even in single crystals [26]. For polycrystalline samples the compositional variations will be at least as pronounced. This means that the magnetic transition becomes spread out over a range of critical temperatures assumed to depend on the local composition, $T_{c}=T_{c}(\mathbf{r})$. If the spread in critical temperatures is comparable to the size of the critical region, it becomes difficult to assign definite critical exponents to the system. This is also true if one tries to determine the universality class from the critical behavior of the specific heat, which has been suggested as a better alternative [27]. Here we will show that direct measurements of the isothermal entropy change not only allows for a reliable determination of the critical temperature but also gives insight into the spin-lattice coupling of the system. The isothermal entropy change, $\Delta S$, is defined as the change in entropy as the magnetic field is changed from 0 to $H$ at a fixed temperature $T$ : $\Delta S(T, H)=S(T, H)-S(T, 0)$. In most systems, including the manganites, it is negative, since the effect of the external field is to order the magnetic moments. The field dependence of $\Delta S$ is characterized by an approximate power-law behavior. Thus, one can define a scaling exponent $b$ through $\Delta S \sim a H^{b}$. The scaling exponent will in general be a function of both $T$ and $H$, making its introduction more a matter of convenience than of profound theoretical justification. Considered as a function of $T, b$ will have a minimum both at $T_{c}$ and at the temperature at which $-\Delta S$ peaks [28]. These two temperatures are in general close together and as $H \rightarrow 0$ they coincide. This means that the critical temperature can be experimentally determined as the temperature for which the scaling exponent is a minimum for low fields.

Six samples with a nominal composition of $\mathrm{La}_{0.67} \mathrm{Ca}_{0.33-x} \mathrm{Sr}_{x} \mathrm{Mn}_{1.05} \mathrm{O}_{3} \quad$ (LCSMO), with $x$ varying from 0.0375 to 0.0750 in steps of 0.0075 , were produced in the form of irregularly shaped pieces of thin plates (thickness $0.3 \mathrm{~mm}$ ) by tape-casting the powder suspended in a slurry, followed by sintering (for details, see Ref. [29]). The slight over-stoichiometry of Mn was chosen to improve the sintering properties of the plates; this does not change the structure or critical temperature [30]. An additional seventh sample with a composition of $\mathrm{La}_{0.67} \mathrm{Ca}_{0.33} \mathrm{MnO}_{3}$ (LCMO) was prepared by the solid-state route. X-ray diffraction shows single-phase samples, and lattice parameters are consistent with those found in the literature. The isothermal entropy change $\Delta S$ was measured using a custom-built differential

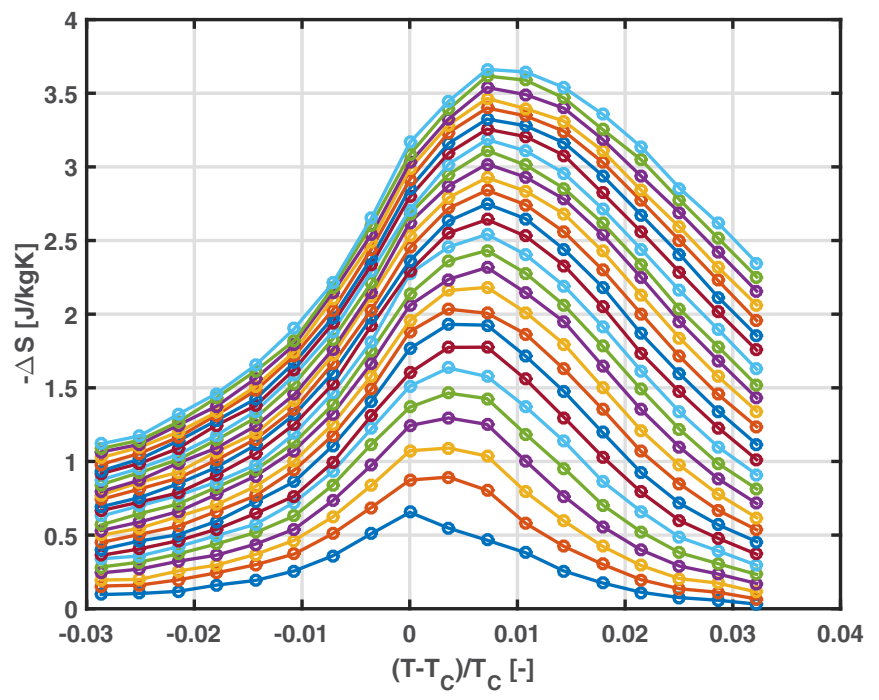

FIG. 1. The isothermal entropy change of $\mathrm{La}_{0.67} \mathrm{Ca}_{0.33-x} \mathrm{Sr}_{x}$ $\mathrm{Mn}_{1.05} \mathrm{O}_{3}$ for $x=0.0375$ for a field change of $0.05-1.5 \mathrm{~T}$, as measured by in-field calorimetry. The relative uncertainty on each data point is approximately $5 \%$.

scanning calorimeter (DSC) equipped with a permanent magnet array allowing the field to be varied [31,32]. This way of determining the isothermal entropy change $\Delta S$ at a fixed temperature, instead of the conventional method of integrating magnetization data [33], allows for a much better temperature resolution of the data, critical for the results presented here. The samples were mounted in such a way as to minimize the geometrical demagnetization. In addition, the measurements were corrected for demagnetization using magnetization data taken in a Lake Shore 7407 vibrating sample magnetometer (VSM) and the geometrical demagnetization correction for a prism [34]. For each sample the isothermal entropy change was measured starting from zero field for applied magnetic fields in the range of $\mu_{0} H=0.05 \mathrm{~T}$ to $1.5 \mathrm{~T}$ with a field step of $0.05 \mathrm{~T}$ and a temperature step of $1 \mathrm{~K}$. A representative set of measurements is shown in Fig. 1. To extract a (temperature-dependent) scaling exponent $b$, the results were fitted to a power-law function of the form $a H^{b}$.

The (mean) critical temperature of each sample, found as the position of the minimum of the scaling exponent $b$, shows a dependence on nominal composition consistent with that previously found in the literature [14], see Fig. 2, confirming the accuracy of the nominal compositions of the samples.

In Fig. 3 we show the scaling exponent at the critical temperature of each sample. A clear trend is visible, showing $b$ decreasing continuously from a value close to $2 / 3$, which is that expected for a pure mean-field model, as the $\mathrm{Sr}$ content $x$ is decreased. All of the LCSMO samples are second order, and the continuous dependence of the scaling exponent on $x$ demonstrates the nonuniversal nature of the field dependence of the isothermal entropy change. It can also be seen that the scaling of $\mathrm{La}_{0.67} \mathrm{Ca}_{0.33} \mathrm{MnO}_{3}$ is markedly different; this is a signature of a more first-order-like phase transition, as will be discussed below.

To interpret these results we use a mean-field approach. Previous theoretical work has been based on a Landau 


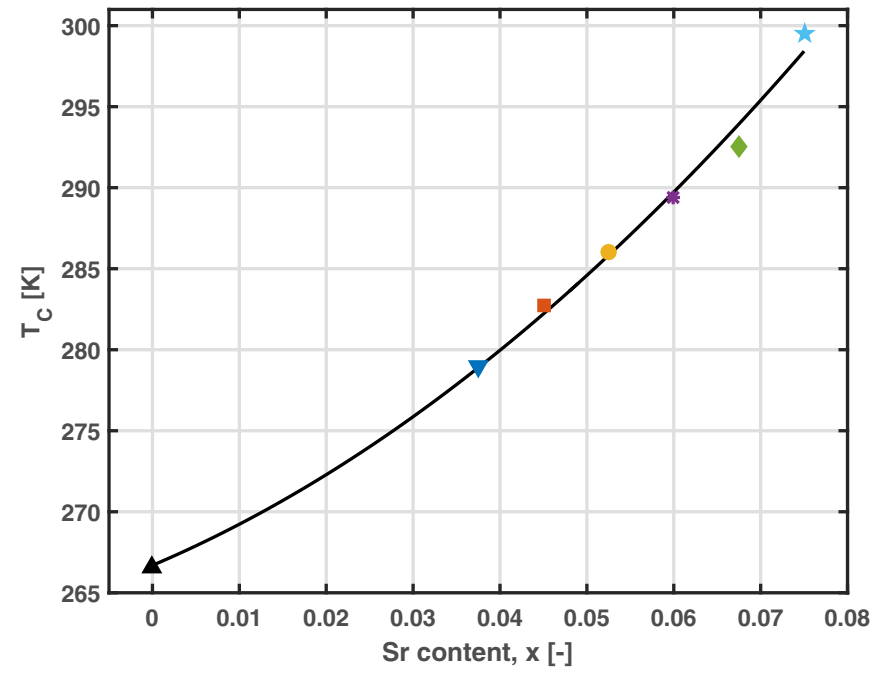

FIG. 2. Critical temperatures of the seven samples as a function of strontium content. The critical temperature was determined as the position of the minimum of the scaling exponent associated with the isothermal entropy change for low fields. The line is a guide to the eye.

expansion of the free energy in which a distribution of $T_{c}$ has been introduced [35,36]. However, the Landau expansion is only valid for small applied fields $H$ and close to $T_{c}$ (being a series expansion in $M$ ); in particular, it does not account for the approach to saturation. It is preferable to consider a microscopic model that accounts for saturation and that gives physically reasonable results for any $T$ and $H$. Here we use the Bean-Rodbell model, which is basically a mean-field model in which the exchange constant depends on the lattice spacing [37]. It is well known that the critical exponents of the Bean-Rodbell model (for the range of parameters where the transition is second order) are those of mean-field theory. However, the model is not identical to Landau theory outside

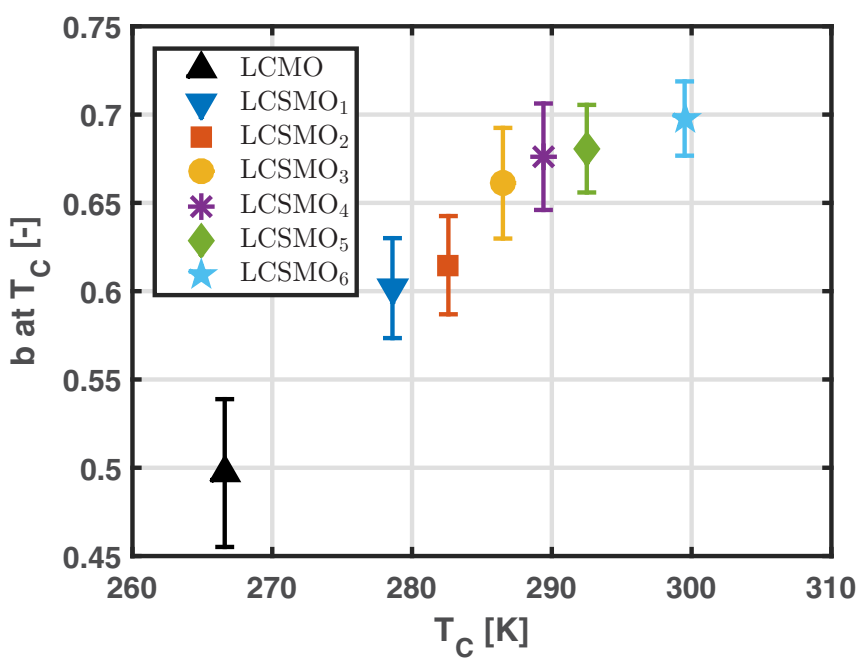

FIG. 3. Experimentally determined scaling exponent of the isothermal entropy change at the critical temperature of each sample and a field change of $1.5 \mathrm{~T}$. For a pure mean-field model with no spin-lattice coupling, a scaling exponent close to $2 / 3$ is expected. the critical region (small reduced temperature and small field). While the Bean-Rodbell model like all mean-field models predicts the wrong critical exponents for the manganites, the spread in $T_{c}$ combined with the limited temperature resolution of most magnetization measurements make it questionable whether the critical region is, in fact, accessed experimentally. Outside the critical region (but still close to $T_{c}$ ), it is well accepted that the Bean-Rodbell model accounts qualitatively and to some extent also quantitatively for the behavior of many magnetocaloric materials, in particular the manganites [38]. As we show below, the field behavior of the isothermal entropy change can in fact be modelled rather satisfactorily by the Bean-Rodbell model. This allows information on the spin-lattice interactions to be extracted.

The Bean-Rodbell model is a mean-field model in which the exchange coupling $\lambda$ is assumed to depend linearly on the interspin distance: $\lambda=\lambda_{0}\left(1+\beta \frac{V-V_{0}}{V_{0}}\right)$, where $V$ is the actual volume, $V_{0}$ the volume in the absence of exchange interactions, and $\beta$ a parameter which controls the strength of the spin-lattice coupling; $\lambda_{0}$ is the value of the exchange coupling corresponding to $V=V_{0}$. The behavior of the model can conveniently be classified by another parameter $\eta$, given by

$$
\eta=40 \frac{N_{s}}{V} \kappa\left(k_{B} T_{0}\right) \beta^{2} \frac{[J(J+1)]^{2}}{(2 J+1)^{4}-1} .
$$

Here $J$ is the magnitude of the spins (in units of $\hbar$ ), $N_{s}$ the number of spins, $\kappa$ the isothermal compressibility, and $T_{0}$ the transition temperature for $\beta=0 ; k_{B}$ is Boltzmann's constant. The order of the phase transition described by the Bean-Rodbell model is determined by $\eta: \eta>1$ correspond to a first order transition. For $\eta<1$ the Bean-Rodbell model describes a second-order phase transition with the same critical exponents as the standard mean-field model but with different behavior at finite fields. This leads to a scaling behavior of $\Delta S$ that depends on $\eta$, as shown recently [12]. To account for compositional variations we introduce a statistical spread in $T_{0}$; i.e., we assume $T_{0}$ to be normally distributed around its average with a standard deviation of $\sigma_{T_{c}}$. We do a least-squares fit of the field dependence of $\Delta S$ at $T=T_{c}$ for each sample, using $\eta$ and $\sigma_{T_{c}}$ as the fitting parameters. This results in the values of $\eta$ (and thus of the magnitude of the spin-lattice interaction $\beta$ ) and $\sigma_{T_{c}}$ shown in Figs. 4 and 5 ; the error bars in the plots reflect a $10 \%$ increase in the residual of the fitting.

The five LCSMO samples all have approximately the same spread, independent of $x$, consistent with the fact that they are produced by the same route, which presumably introduces similar amounts of compositional variation. The spread in critical temperature is in all cases of the order of $1-1.5 \mathrm{~K}$, while the spread in $T_{c}$ for the LCMO sample is slightly higher. The value of $\eta$ increases as $x$ decreases, i.e., addition of strontium decreases the magnitude of the spin-lattice coupling. For the LCMO sample we find a value of 1.2 for $\eta$, meaning that the transition in LCMO is of first order. This is consistent with several other reports in the literature [14-16]. Our finding that the magnitude of the spin-lattice interaction increases monotonously with decreasing $x$ quantifies the often-stated assertion that the LCSMO materials become more first-orderlike as $x=0$ is approached. The fact that $\eta$ is significantly 


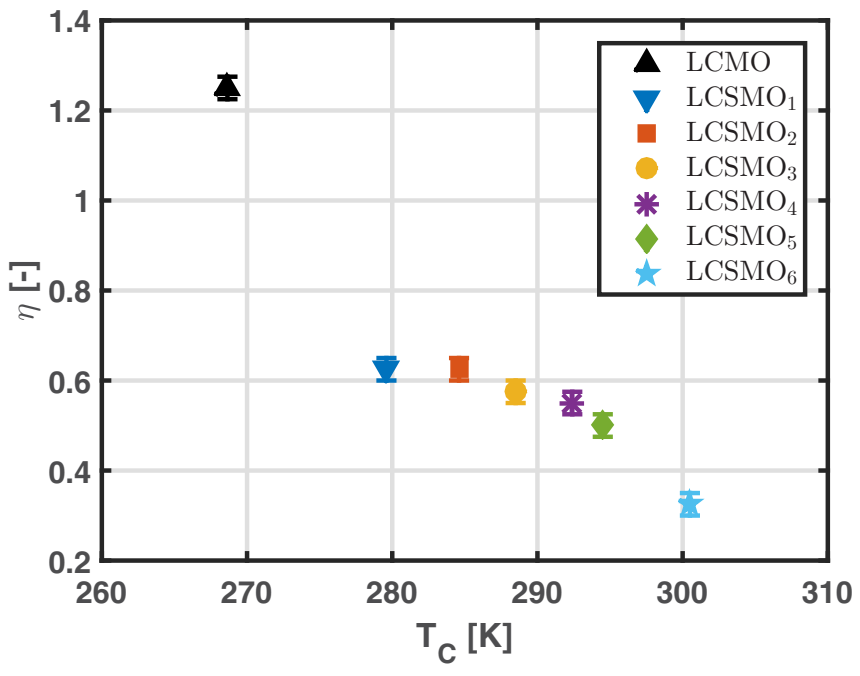

FIG. 4. Bean-Rodbell parameter $\eta$ extracted from the experimental field dependence of $\Delta S$ by fitting to the Bean-Rodbell model with a spread in critical temperatures. The increase in $\eta$ as the strontium content decreases is equivalent to an increase in the spin-lattice coupling. A value $\eta>1$ corresponds to a first-order transition.

larger than 1 for $x=0$ suggests that the first-order transition may extend to finite, but small $x$ (certainly smaller than $x=0.0375$ which we unequivocally identify as second order). We see that even though there are no critical exponents associated with a first-order transition, our approach looking at the full field dependence of $\Delta S$ allows us to identify the order of the transition.

In conclusion, we have demonstrated experimentally that the finite-field scaling of the magnetocaloric effect close to a second-order phase transition is not universally determined by the critical exponents of the transition. Further, we have

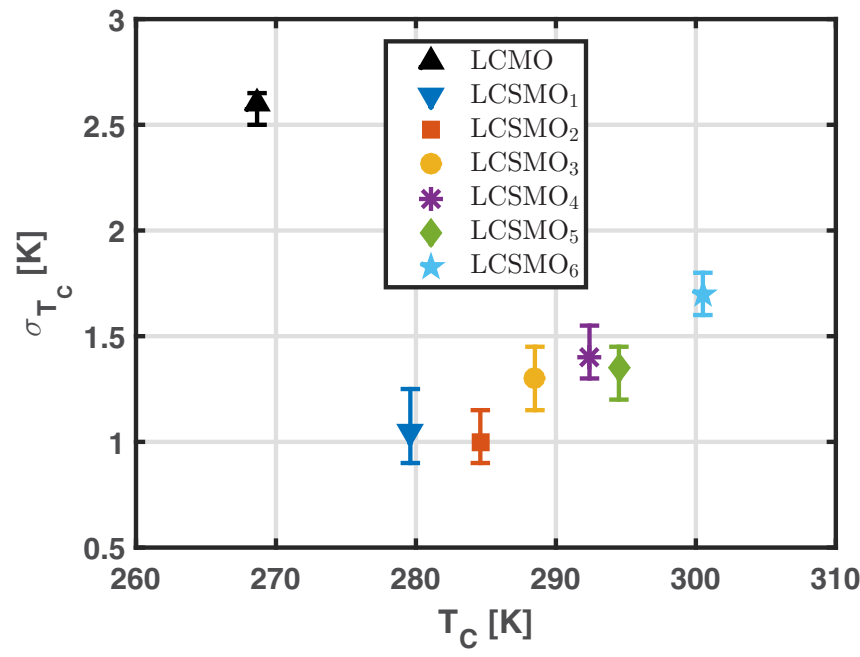

FIG. 5. The spread in critical temperature as measured by the standard deviation $\sigma_{T}$, calculated from a fit of the model to the experimental field dependence of $\Delta S$ at $T_{c}$.

argued that compositional variations in many cases lead to a spread in $T_{c}$ of a magnitude that precludes direct access to the critical region. Nevertheless, a careful determination of the field dependence of the isothermal entropy change close to the average transition temperature allowed us to determine the variation of the effective spin-lattice coupling as a function of the strontium content of lanthanum calcium strontium manganite. It will be of interest to apply this analysis to different series of magnetocaloric compositions.

This work was in part financed by the ENOVHEAT project, which is funded by Innovation Fund Denmark (Contract No. 12-132673). The assistance of Kjeld Bøhm Andersen in the tape-casting of the samples is gratefully acknowledged.
[1] J. Coey, M. Viret, and S. von Molnar, Adv. Phys. 48, 167 (1999).

[2] M. B. Salamon and M. Jaime, Rev. Mod. Phys. 73, 583 (2001).

[3] N. Mathur and P. Littlewood, Phys. Today 56(1), 25 (2003).

[4] M. H. Phan and S. C. Yu, J. Magn. Magn. Mater. 308, 325 (2007).

[5] L. I. Koroleva, D. M. Zashchirinskii, A. S. Morozov, and R. Szymczak, J. Exp. Theor. Phys. 115, 653 (2012).

[6] X. Moya, C. Ducati, L. Phillips, M. Ghidini, M. Vickers, E. Defay, N. Mathur, L. Hueso, O. Hovorka, A. Berger, L. Hueso, F. Maccherozzi, S. Dhesi, A. Tovstolytkin, D. Podyalovskii, M. Ghidini, and E. Defay, Nat. Mater. 12, 52 (2013).

[7] A. Smith, C. R. H. Bahl, R. Bjørk, K. Engelbrecht, K. K. Nielsen, and N. Pryds, Adv. Energy Mater. 2, 1288 (2012).

[8] P. Weiss and A. Piccard, J. Phys. Theor. Appl. 7, 103 (1917).

[9] A. Smith, Eur. Phys. J. H 38, 507 (2013).

[10] V. Franco and A. Conde, Int. J. Refrig. 33, 465 (2010).

[11] V. Franco, A. Conde, J. M. Romero-Enrique, Y. I. Spichkin, V. I. Zverev, and A. M. Tishin, J. Appl. Phys. 106, 103911 (2009).
[12] A. Smith, K. K. Nielsen, and C. R. H. Bahl, Phys. Rev. B 90, 104422 (2014).

[13] A. R. Dinesen, S. Linderoth, and S. Mørup, J. Phys.: Condens. Matter 17, 6257 (2005).

[14] J. Mira, J. Rivas, F. Rivadulla, C. Vázquez-Vázquez, and M. A López-Quintela, Phys. Rev. B 60, 2998 (1999).

[15] J. Mira, J. Rivas, F. Rivadulla, and M. Quintela, Phys. B Condens. Matter 320, 23 (2002).

[16] V. Amaral, J. Araujo, Y. Pogorelov, J. Sousa, P. Tavares, J. Vieira, P. Algarabel, and M. Ibarra, J. Appl. Phys. 93, 7646 (2003).

[17] K. Morrison, A. Berenov, and L. Cohen, Mater. Res. Soc. Symp. Proc. 1310, 31 (2011).

[18] A. Oleaga, A. Salazar, D. Prabhakaran, and A. T. Boothroyd, J. Appl. Phys. 95, 7366 (2004).

[19] A. Oleaga, A. Salazar, D. Prabhakaran, and A. T. Boothroyd, Phys. Rev. B 70, 184402 (2004).

[20] M. Nasri, M. Triki, E. Dhahri, and E. K. Hlil, J. Alloys Compd. 546, 84 (2013). 
[21] M. H. Phan, V. Franco, N. S. Bingham, H. Srikanth, N. H. Hur, and S. C. Yu, J. Alloys Compd. 508, 238 (2010).

[22] J. L. García-Muñoz, J. Fontcuberta, M. Suaaidi, and X. Obradors, J. Phys.: Condens. Matter 8, L787 (1996).

[23] A. R. Dinesen, Magnetocaloric and Magnetoresistive Properties of $\mathrm{La}_{0.67} \mathrm{Ca}_{0.33-x} \mathrm{Sr}_{x} \mathrm{MnO}_{3}$, Ph.D. thesis, Technical University of Denmark, 2004.

[24] S. Kaul, J. Magn. Magn. Mater. 53, 5 (1985).

[25] M. H. Phan, G. T. Woods, A. Chaturvedi, S. Stefanoski, G. S. Nolas, and H. Srikanth, Appl. Phys. Lett. 93, 252505 (2008).

[26] N. G. Bebenin, R. I. Zainullina, and V. V. Ustinov, J. Magn. Magn. Mater. 322, 963 (2010).

[27] A. Gamzatov, A. Aliev, K. Khizriev, I. Kamilov, and A. Mankevich, J. Alloys Compd. 509, 8295 (2011).

[28] V. Franco, A. Conde, M. D. Kuz'min, and J. M. RomeroEnrique, J. Appl. Phys. 105, 07A917 (2009).

[29] R. Bulatova, C. R. H. Bahl, K. Andersen, L. T. Kuhn, and N. Pryds, Int. J. Appl. Ceram. Tec. 12, 891 (2015).
[30] I. Biering, M. Menon, and N. Pryds, in Advances in Energy Materials: Ceramic Transactions, edited by F. Dogan and N. Manjouran (Wiley, New Jersey, 2009), Vol. 205.

[31] K. K. Nielsen, H. N. Bez, L. von Moos, R. Bjørk, D. Eriksen, and C. R. H. Bahl, Rev. Sci. Instrum. 86, 103903 (2015).

[32] R. Bjørk, C. R. H. Bahl, A. Smith, and N. Pryds, J. Magn. Magn. Mater. 322, 3664 (2010).

[33] V. K. Pecharsky and K. A. Gschneidner Jr., J. Appl. Phys. 86, 565 (1999).

[34] A. Aharoni, J. Appl. Phys. 83, 3432 (1998).

[35] J. Lyubina, M. D. Kuz'min, K. Nenkov, O. Gutfleisch, M. Richter, D. L. Schlagel, T. A. Lograsso, and K. A. Gschneidner, Phys. Rev. B 83, 012403 (2011).

[36] N. G. Bebenin, R. I. Zainullina, and V. V. Ustinov, J. Appl. Phys. 113, 073907 (2013).

[37] C. P. Bean and D. S. Rodbell, Phys. Rev. 126, 104 (1962).

[38] J. A. Turcaud, A. M. Pereira, K. G. Sandeman, J. S. Amaral, K. Morrison, A. Berenov, A. Daoud-Aladine, and L. F. Cohen, Phys. Rev. B 90, 024410 (2014). 